\title{
Presença da família da criança nas situações de emergência e procedimentos invasivos: revisão integrativa
}

\author{
Child family presence in emergencies and invasive procedures: integrative review \\ Presencia de la familia de niños en situaciones de emergencia y procedimientos invasivos: \\ revisión integrativa
}

\author{
Leonardo Aparecido Motta Mendes ${ }^{1}$, Ana Paula Dias França Guareschi
}

\section{Resumo}

Objetivo: Identificar a importância da opção da presença da família nas situações de emergência e procedimentos invasivos e as limitações para efetivação desta prática.

Métodos: Revisão integrativa, no período de 2010 a 2018, nas bases de dados LILACS, Medline, PubMed e SciELO, analisados 36 artigos de acordo com os critérios de inclusão.

Resultados: Elaboradas categorias empíricas, 1- importância da opção da presença da família nas situações de emergência e procedimentos invasivos (35 artigos), com as unidades de significado, família como fonte de apoio, compreensão da importância da família pela equipe de saúde e comunicação da enfermagem com a família e 2- limitações para a efetivação da opção da presença da família nas situações de emergência e procedimentos invasivos (26 artigos), com as subcategorias hegemonia da equipe e insegurança/despreparo profissional.

Conclusão: A presença da família nos procedimentos invasivos e situações de emergências deve ser considerada pela equipe de saúde, pois contribui na assistência à criança hospitalizada.

\begin{abstract}
Objective: To identify the importance of the option of family presence in emergencies and invasive procedures and the limitations to the effectiveness of this practice.

Methods: Integrative review, in the period from 2010 to 2018, in the LILACS, Medline, PubMed and SciELO databases; 36 articles were analyzed according to the inclusion criteria.

Results: Elaborated empirical categories: 1- importance of the option of family presence in emergencies and invasive procedures ( 35 articles), with units of meaning, family as source of support, understanding the importance of the family by the health team and nursing communication with family; 2 - limitations to the effectiveness of the option of family presence in emergencies and invasive procedures (26 articles), with the subcategories hegemony of the team and professional insecurity / unpreparedness.

Conclusion: The health team should consider the presence of the family in the invasive procedures and emergencies, as it contributes to the care of hospitalized children.
\end{abstract}

\section{Resumen}

Objetivo: Identificar la importancia de la opción de la presencia de la familia en las situaciones de emergencia y procedimientos invasivos y las limitaciones para la efectividad de esta práctica.

Métodos: Revisión integrativa, en el período de 2010 a 2018, en las bases de datos LILACS, Medline, PubMed y SciELO, analizados 36 artículos de acuerdo con los criterios de inclusión.

Resultados: Elaboradas categorías empíricas: 1- importancia de la elección de la familia en presencia de emergencias y procedimientos invasivos (35 artículos), con las unidades de significados familia como una fuente de apoyo, la comprensión de la importancia de la familia por el equipo de salud y la comunicación de la enfermería con la familia y 2 - limitaciones para la efectividad de la opción de la presencia de la familia en las situaciones de emergencia y procedimientos invasivos (26 artículos), con las subcategorías hegemonía del equipo e inseguridad/ despreparo profesional.

Conclusión: La presencia de la familia en los procedimientos invasivos y situaciones de emergencias debe ser considerada por el equipo de salud, pues contribuye en la asistencia al niño hospitalizado.

\section{Descritores}

Enfermagem pediátrica; Emergência; Família; Ressuscitação; Criança

Keywords

Nursing pediatric; Emergency; Family; Ressuscitation; Child

\section{Descriptores}

Enfermería pediátrica; Emergencia;

Familia; Resucitación; Niño

\section{Como citar:}

Mendes LA, Guareschi AP. [Child family presence in emergencies and invasive procedures: integrative review]. Rev Soc Bras Enferm Ped. 2018;18(2):96-102. Portuguese

${ }^{1}$ Centro Universitário São Camilo, Membro, São Paulo, SP, Brasil.

2 Escola Paulista de Enfermagem, Universidade Federal de São Paulo, São Paulo, SP, Brasil.

Conflitos de interesse: nada a declarar.

Submissão: 28 de Julho de 2016 | Aceite: 27 de Junho de 2019

Autor correspondente: Ana Paula Dias França Guareschi | Rua Napoleão de Barros, 754, 04024-002, São Paulo, SP, Brasil. guareschi@unifesp.br

https://orcid.org/0000-0003-2739-3118

DOI: http://dx.doi.org/10.31508/1676-3793201800015 


\section{Introdução}

O Cuidado Centrado no Paciente e Família (CCPF) tem sido difundido através das pesquisas, como um cuidado ideal a ser aplicado por profissionais, que atuam na assistência aos pacientes e às suas famílias, nos diversos serviços de saúde. Os profissionais da enfermagem são os que mais convivem com a família, com isso, nos últimos anos muitas pesquisas sobre a atuação da equipe de enfermagem junto à família foram divulgadas. ${ }^{(1-3)}$

$\mathrm{O}$ CCPF tem sido uma abordagem inovadora para o planejamento, execução e avaliação dos cuidados de saúde, que se baseia em uma parceria, mutuamente, benéfica entre os pacientes, famílias e profissionais, tendo como o objetivo promover a saúde e o bem-estar dos indivíduos e família e restaurar seu controle e dignidade. $^{(4)}$

A criança necessita de uma atenção especial, dadas as peculiaridades biológicas e psicológicas, e diversos fatores que a colocam em situação de risco, essa filosofia de cuidado promove a participação da família e a reconhece como parceira no cuidado, estimulando sua vinculação afetiva com o paciente, aspecto necessário para promover seu desenvolvimento físico e emocional. Devido as particularidades da criança, as principais demandas dos serviços de emergência pediátricos são doenças respiratórias, doenças do trato digestivo, os estados convulsivos, intoxicações, acidentes e traumas, que podem levar estes pacientes a uma parada cardiorrespiratória, que constitui a emergência médica de maior importância na área pediátrica. ${ }^{(1,5)}$

Nos locais de atendimento multidisciplinar à criança crítica, como o Pronto Socorro Infantil (PSI), Unidade de Terapia Intensiva Pediátrica (UTIP) e Unidade de Terapia Intensiva Neonatal (UTIN), tem-se como pressuposto que os profissionais devem atuar com competência, habilidades e atitudes compatíveis para as situações emergenciais, pois o foco do cuidado é salvar vidas e restaurar a saúde da criança, através dos procedimentos invasivos e emergenciais, nestes cenários a participação da família ainda é um paradigma a ser explorado.

O CCPF e o Estatuto da Criança e do Adolescente ${ }^{\left({ }^{(6)}\right.}$ respaldam o cuidado e permanência da família junto à criança, porém ainda na prática clínica, nos momentos de emergência ou procedimentos invasi- vos, existem lacunas na execução destes princípios. Apesar da vasta literatura sobre o CCPF e a expressiva publicação internacional sobre a participação da família em situações de emergência e procedimentos invasivos, ainda há escassez destas pesquisas no Brasil.

O estudo se propõe a identificar a importância da opção da presença da família nas situações de emergência e procedimentos invasivos e as limitações para efetivação desta prática.

\section{Métodos}

Para o desenvolvimento deste estudo, adotou-se o método da revisão integrativa da literatura, que busca a exaustão dos estudos sobre o tema investigado, para se obter um profundo entendimento de determinado fenômeno, baseando-se em estudos anteriores. Isso permite incorporar as evidências na prática, a fim de reunir e sintetizar os resultados de pesquisas sobre uma questão delimitada, de maneira sistemática e ordenada, além de aprofundar o conhecimento do tema investigado. Uma revisão dá suporte para a tomada de decisão e a melhoria da prática, possibilitando a síntese do estado de conhecimento acerca de determinado assunto. Podese fazer uma análise ampla da literatura, contribuindo para discussões sobre métodos e resultados de pesquisas, além de apontar lacunas no conhecimento que precisam ser preenchidas com a realização de novos estu$\operatorname{dos}^{(7)}$. O estudo foi dividido nas seguintes etapas:

$1^{\text {a }}$ Etapa (Definição da pergunta norteadora): etapa de maior importância, pois conduziu o desenvolvimento da revisão integrativa, dessa forma, a pergunta norteadora da presente revisão foi: Quais são as evidências científicas sobre a importância da opção da presença da família, nas situações de emergência e procedimentos invasivos e as limitações para efetivação desta prática.

$2^{2}$ Etapa (Busca ou amostragem na literatura): esta etapa consistiu na procura em bases eletrônicas e na submissão dos achados aos critérios de inclusão e exclusão, dos artigos do período de 2010 a 2018. A busca foi realizada de acordo com a estratégia PICO (Patient Intervention Control Outcome) utilizando os descritores dos sistemas de metadados: Medical Subject Headings (MeSH) e Descritores em Ciências da Saúde (DECs), com os operadores booleanos AND e 
OR, conforme termos a seguir: Enfermagem Pediátrica, Emergência, Família, Ressuscitação, Criança.

$3^{\mathrm{a}}$ Etapa (Coleta de dados): momento para extrair os dados das publicações. Para esta etapa foram utilizadas pesquisas pertencentes às seguintes bases de dados: Literatura Internacional em Ciências da Saúde (PubMed), da Scientific Electronic Library Online (SciELO) e da Literatura Latino-Americana e do Caribe em Ciências da Saúde (Lilacs) e Medical Literature Analysis and Retrieval System Online (Medline). Os artigos foram identificados nas bases de dados e analisados pelo título e resumo de forma independente e simultânea, e aos pares. Os critérios de inclusão utilizados foram trabalhos: 1- Indexados nas bases de dados citadas; 2- Escritos nos idiomas português, inglês e espanhol; 3- Publicados nos últimos 18 anos; 4- Com texto completo disponível e gratuito; 5Cenário de unidades hospitalares críticas e de emergências. A exclusão de artigos abrangeu trabalhos que não apresentassem resumos na íntegra nas bases de dados e os que relacionaram a temática do estudo no contexto pré-hospitalar e centro cirúrgico.

$4^{a}$ Etapa (Análise crítica dos estudos incluídos e categorização dos estudos selecionados): esta etapa compreendeu a análise rigorosa dos títulos, resumos e palavras-chave dos 63 artigos obtidos com a estratégia de busca para que fossem aplicados os critérios de inclusão. Mediante nova leitura, 27 artigos foram excluídos: porque o texto completo não correspondia ao tema proposto e pela duplicidade de artigos nas bases de dados. Depois de sintetizadas e categorizadas as informações colhidas dos artigos científicos selecionados anteriormente, 36 artigos foram selecionados e organizados ao longo do estudo, baseando-se nas semelhanças entre os temas abordados por eles. As categorias de interesse foram organizadas em um instrumento de coleta criado pelos pesquisadores com dados sobre título, autores, referências e ano de publicação. Os artigos foram lidos e analisados na íntegra para extrair modalidade de estudo, importância da opção da presença da família nas situações de emergências e procedimentos invasivos e as limitações para sua efetivação.

5a Etapa (Discussão dos resultados): nesta etapa procedeu-se a leitura e comparação dos artigos, levando à discussão dos estudos analisados na revisão integrativa, buscando atingir os objetivos do estudo. Destaca-se que não foi realizada nenhuma análise estatística quantitativa dos estudos (metanálise).

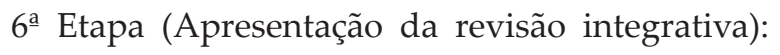
nesta etapa foi apresentada a interpretação dos resultados obtidos por meio da revisão integrativa.

\section{Resultados}

Foram representados nos quadros 1 e 2, os elementos de essencialidade dos artigos que retrataram a importância da opção da presença da família, em situações de emergências/procedimentos e as limitações dessa prática. As revistas que mais publicaram artigos sobre a temática deste estudo: J Emerg Nurs (3 artigos); Arch Dis Child, Am J Crit Care, Int Emerg Nurs, Rev Bras de Enf, Cien Cuid Salud, Rev de Bioét (2 artigos). Os períodos de maior publicação foram os anos de 2011 (8 artigos), 2013 (7 artigos), 2014 (6 artigos), 2012 e 2014 (6 artigos cada) (Quadro 1). No quadro 1 são apresentados os elementos que retratam a importância da opção da presença da família nas situações de emergência e procedimentos invasivos. Foi identificado em 14 artigos a comunicação da enfermagem com a família, em 12 artigos a família como fonte de apoio e em 9 artigos a compreensão da importância da família pela equipe de saúde, o menor número de artigos sobre esta temática, está atrelado a escassez de estudos que investiguem mais a percepção dos profissionais de saúde.

Quadro 1. Importância da opção da presença da família nas situações de emergência e procedimentos invasivos

\begin{tabular}{l} 
Elementos que retratam a importância da opção da presença da família nas \\
situações de emergência e procedimentos invasivos \\
\hline Família como fonte de apoio $^{(1,8-19)}$ \\
\hline Compreensão da importância da família pela equipe de saúde \\
\hline Comunicação da enfermagem com a família(1,1,8-10,12,1618,19,26-30) \\
\hline
\end{tabular}

No quadro 2 são apresentados os elementos que descrevem as limitações da efetivação da opção da presença da família, nas situações de emergência e procedimentos invasivos. Foi identificado em 17 artigos a descrição da insegurança e despreparo profissional e em 9 artigos a hegemonia da equipe de saúde.

Quadro 2. Limitações para a efetivação da opção da presença da família, nas situações de emergência e procedimentos invasivos

Elementos que descrevem as limitações para efetivação da presença da família nas situações de emergência e procedimentos invasivos

Hegemonia da equipe de saúde ${ }^{(1,14,22,26,27,30-33)}$

Insegurança / Despreparo profissional $\left.\right|^{(11,14,15,17,19,30,31,34-44)}$ 


\section{Discussão}

Os dados resgatados nos artigos selecionados, foram analisados segundo referencial analítico do $\mathrm{CCPF},{ }^{(4)}$ que permitiram a construção das Categorias Empíricas a seguir apresentadas.

\section{Importância da opção da presença da família na realização de procedimentos invasivos e situações de emergência}

A categoria Importância da opção da presença da família nas situações de emergência e procedimentos invasivos é composta pelas unidades de significado: Família como fonte de apoio; Compreensão da importância da família pela equipe de saúde; Comunicação da enfermagem com a família, que apresentam a família como sujeito de cuidado e a percepção e comunicação da equipe de saúde em relação a presença da família nas situações de emergência e procedimentos invasivos.

\section{Família como fonte de apoio}

Existe o consenso da literatura com relação a importância da presença da família no processo de hospitalização da criança, visto que ela deve ser considerada como parte indispensável do processo assistencial, são destacados os benefícios que isso pode acarretar para família, criança e equipe. Nesse contexto, a prática de enfermagem com famílias voltada para ações de orientação e busca de informações, propicia o empoderamento das famílias em desenvolver o cuidado à criança. Por isso, reconhecem e valorizam o componente educativo do cuidado atribuído pela equipe de saúde, muitas delas se sentem estimuladas a cuidar, auxiliando em procedimentos e na tomada de decisões com a equipe, quando os profissionais da enfermagem esclarecem suas dúvidas em relação à doença da criança, bem como, quando recebem orientações que permitem aprimorar o cuidado prestado à elas. ${ }^{(8,10-11,16)}$

Nessa abordagem, o recém-nascido, a criança, o adolescente, passam a ser compreendidos como integrantes de uma família e também como foco do cuidado da equipe de saúde. Nesse cenário, a família é reconhecida como cliente que necessita de apoio, ao mesmo tempo em que é estimulada a cuidar de seu membro, além de elemento colaborador de uma assistência planejada em conjunto. ${ }^{(17)}$

A proposta desta filosofia de cuidado já está comprovada como benéfica para a criança e família, o desafio atual está atrelado a sua aplicabilidade em todos os serviços de saúde brasileiros, em especial na área neonatal. Outro avanço da proposta discutida na $5^{\text {a }}$ Conferência Internacional do Instituto de Atendimento Centrado no Paciente e na Família (IPFCC) de reconhecer os membros da família como parte da equipe de saúde, contribuindo com sua expertise no planejamento da assistência interprofissional à criança. ${ }^{(13)}$

Para a família apoiar a criança nos procedimentos invasivos e situações de emergência, primeiramente, precisa ser garantida sua presença, no PSI, UTIP e UTIN, conforme o respaldo da Lei no. 13.257,(6) que regulamenta o Estatuto da Criança e do Adolescente, dispõe no seu Artigo 12, que: “[...] os estabelecimentos de atendimento à saúde, inclusive as unidades neonatais, de terapia intensiva e de cuidados intermediários, deverão proporcionar condições para a permanência em tempo integral de um dos pais ou responsável, nos casos de internação de criança ou adolescente".

Outro aspecto a ser analisado pela equipe de saúde é a vulnerabilidade, presente nas famílias. Estudos apontam que apesar das constantes manifestações de descontentamento, a família se submete ao que lhe é imposto, intensificando-se o sentimento de vulnerabilidade. A solidão e o isolamento na experiência do familiar, a sobrecarga física e emocional são constantes, principalmente, em momentos críticos e por não conseguir suportar o peso da situação da criança. Estudos apontam o efeito negativo da ausência dos pais durante as situações de emergência, na elaboração do processo de luto, ${ }^{(1,12,18,19)}$ A fim de modificar essa realidade e contribuir para que as famílias se sintam cuidadas durante a vivência dessa experiência, cabe a equipe de saúde implementar ações que promovam a presença da família durante procedimentos invasivos e situações de emergência, em unidades de cuidados críticos pediátricos e neonatais e pronto socorro infantil.

\section{Compreensão da importância da família pela equipe de saúde}

Para alguns profissionais a família revela-se como um ser importante de direitos garantindo a sua contribui- 
ção na hospitalização da criança. No hospital, a família favorece a troca de informações, auxilia a tornar os procedimentos mais aceitáveis a criança, favorece a prevenção de acidentes e a terapêutica instituída, propiciando que esta receba um atendimento humanizado e acolhedor. ${ }^{(20-23)}$ A presença da família é essencial, pois "são os porta-vozes da criança, representam os sentimentos, as atitudes, os comportamentos socioculturais internalizados no mundo da vida da criança. São os mediadores da criança no mundo do hospital", onde a partir desse conhecimento, pode-se propor a construção de guias e protocolos para nortear a prática dos profissionais da equipe de saúde, contemplando a família como participante ativa nesse processo..$^{(1,9,24,25)}$

\section{Comunicação da enfermagem com a família}

Estudos apontam que os pais consideram importante, serem informados e presenciarem as situações em que a criança é submetida durante a hospitalização, principalmente, as emergenciais. No momento crítico, a família fornece subsídios para efetivação da assistência emergencial, sendo inquestionável, a sua contribuição nas situações em que não há como detectar o diagnóstico precoce na emergência. No entanto, para um atendimento integral não podem ser desconsideradas suas necessidades e fragilidades, pois assim como a criança, a família também necessita ser amparada afetivamente e sentir-se cuidada. ${ }^{(1,10,26,27)}$ Outro aspecto descrito nos estudos é a necessidade da família vivenciar o atendimento de emergência, tendo a ciência do que está sendo realizado com sua criança, evitando o cenário angustiante de estar fora da sala de emergência, aguardando notícias sobre o estado clínico do filho, podendo gerar dúvidas sobre o atendimento que está sendo prestado e se a equipe de saúde fez o que era necessário para salvar seu filho. ${ }^{(1,8,10,18,28)}$ No contexto do atendimento de emergência, estudo mostra que, quando é dada a opção, as famílias em geral escolhem permanecer ao lado da criança, propicia uma comunicação mais efetiva com a equipe multiprofissional. ${ }^{(29)}$ Importante ressaltar que para a efetivação dessa prática, é necessário investimento institucionais de capacitação da equipe para este momento. ${ }^{(12,16)}$ Há consenso dos estudos da necessidade de um profissional permanecer junto com a família durante $o$ atendimento de emergência e procedimentos invasivos, explicando as intervenções em que a criança está sendo submetida. ${ }^{(16,19,30)}$

\section{Limitações para a efetivação da opção da presença da família, nas situações de emergência e procedimentos invasivos}

A categoria Limitações para a efetivação da opção da presença da família, nas situações de emergência e procedimentos invasivos abarca as unidades de significado: Hegemonia da equipe de saúde e Insegurança/Despreparo profissional, que apresentam as dificuldades encontradas nos serviços de emergências e cuidados críticos, na implementação do CCPF na sua integralidade. Esta dificuldade de implementação do CCPF foi descrita na pesquisa, ${ }^{(1)}$ que investigou as ações promotoras da presença da família na RCP e nos Procedimentos Invasivos em unidades de cuidados críticos pediátricos e neonatais. O estudo aponta a necessidade de elaborar uma política institucional declarada, programa de sensibilização e capacitação da equipe de saúde para incluir a família no cuidado e atender suas necessidades.

\section{Hegemonia da equipe de saúde}

Ao entrar para o mundo do hospital, as famílias passam a ser governadas por várias normas e rotinas impostas pelos profissionais, instrumentos estes, muito utilizados pelo profissional de enfermagem, a fim de, redirecionar seu processo de trabalho, e harmonizar as funções dos diversos setores que coabitam no hospital. ${ }^{(26)}$ Nas relações da equipe com as famílias, parece haver uma assimetria de poder e, invariavelmente, a equipe procura impor as famílias suas opiniões, exercendo sobre elas certa dominação. Por sua vez, as famílias possuem capacidades diferentes de negociação e de controle sobre suas próprias vidas. Este fato associado à vulnerabilidade em que se encontram no hospital facilita a submissão das famílias à equipe, e para alguns profissionais resulta em maior controle sobre o território do hospital e sobre as informações acerca do diagnóstico e tratamento da criança, tendo mais condições de impor-se as famílias. No entanto, essa aparente submissão pode não significar uma apatia da família, mas, sim, uma estratégia de enfrentamento possível da situação, em benefício da criança. Al- 
guns profissionais ainda persistem em atuar baseados no modelo biomédico, em que cuidar é reconhecido apenas com o fazer objetivo. A equipe decide quando e como a família deve participar, em procedimentos invasivos e em situações de emergências, restringindo seu espaço para fazer suas escolhas. . $1,14,22,27,31,32)^{2}$

No estudo ${ }^{(30)}$ foi identificado que os profissionais formados há mais tempo, acreditam que a família não deva participar da decisão sobre sua própria permanência na sala de emergência. Em outra pesquisa ${ }^{(33)}$ foi citada a preocupação dos profissionais com os aspectos legais e potenciais riscos envolvidos com a presença da família durante a reanimação pediátrica. Como a família encontra-se fragilizada, pela internação hospitalar do filho, esta, na maioria das vezes, fica à mercê das decisões dos profissionais. Assim, compete ao enfermeiro tomar a iniciativa de integrá-la ao processo de cuidado da criança, na busca por relações menos conflitantes e mais efetivas, no sentido de se propiciar um cuidado mais qualificado, sensível e humano. ${ }^{(22,27)}$

\section{Insegurança/Despreparo profissional}

Outro aspecto importante a limitação da opção da presença da família é a insegurança e desconforto do profissional de saúde, estando a família ao seu lado no atendimento, pois eles referem que se sentem inseguros nas tomadas de decisões nos procedimentos nos quais a criança é submetida. ${ }^{(17,19)}$ Estudo $^{(30)}$ apresenta a opinião dos enfermeiros pediátricos sobre a presença da família na reanimação cardiopulmonar, consideram que os pais têm um efeito negativo sobre a capacidade dos profissionais que prestam assistência à criança, sendo a reanimação cardiopulmonar, o momento de maior conflito para decidirem se permitem ou não a presença dos pais da criança. A percepção que a família interfere na atuação dos profissionais, também foi identificada na literatura. ${ }^{(11,14,30-43)}$

Outro aspecto evidenciado além da insegurança foi o despreparo profissional que pode ser justificado pela lacuna do CCPF na sua formação; escassez de evidências científicas de alta qualidade; ausência de educação permanente dos profissionais dos serviços de emergência e cuidados intensivos e recursos humanos, materiais e estruturais das unidades que dificultam a permanência da família durante as situações de emergência e procedimentos invasivos. ${ }^{(11,19,30,31,35-42)}$

\section{Conclusão}

A presença da família em procedimentos e em situação de emergências é muito importante, pois contribui direta e indiretamente com a assistência à criança hospitalizada, sendo imprescindível que as instituições de saúde possam instituir filosofia do CCPF e fornecer subsídios para se elaborar protocolos assistenciais, que contemplem as necessidades das famílias, para modificar uma prática ainda centrada no indivíduo e avançar em direção a uma que contemple os princípios centrais do modelo do CCPF. É preciso investir também na educação permanente dos profissionais, a fim de reforçar atitudes, comportamentos e valores que promovam a opção da presença e a parceria com a família nos cuidados com a criança, além da ampliação de pesquisas sobre a presença da família durante os procedimentos invasivos e situações de emergência que possam corroborar, para a prática baseada em evidências científicas. Este estudo tem como implicação para enfermagem, respaldar as equipes de saúde hospitalares, com base nas pesquisas analisadas, na efetivação da opção da presença da família nas situações de emergências e procedimentos invasivos.

\section{Referências}

1. Ferreira CA, Balbino FS, Balieiro MM, Mandetta MA. Presença da família durante reanimação cardiopulmonar e procedimentos invasivos em crianças. Rev Paul Pediatr. 2014;32(1):107-13.

2. Jolley J, Shields L. The evolution of family centered care.J Pediatr Nurs. 2009;24(2):164-70.

3. Neves ET, Cabral IE, Silveira A. Rede familial de crianças com necessidades especiais de saúde: implicações para a enfermagem. Rev Lat Am Enfermagem. 2013; 21(2):1-9.

4. Johnson MA, Conway LS, Edgman-Levitan S, Sodomka JS, Ford D. Partnering with patients and families to design a patient-and family-centered health care system: recommendations and promising practices [Internet]. Cambridge: Institute for Family-Centered Care; 2008. [cited 2017 out 17]. Available from: http://www.familycenteredcare.org/pdf/ PartneringwithPatientsandFamilies.pdf.

5. Garcia DS. Crianças atendidas em pronto-atendimento e pronto-socorro: subsídios para melhoria da assistência. Enfermagem em Foco. 2011;2(2):128-32.

6. Brasil. Ministério da Justiça. Lei 13.257 , de 8 de Março de 2016. Estatuto da Criança e do Adolescente. Dispõe sobre a proteção integral à criança e ao adolescente. Brasília; 2016.

7. Sampieri RH, Collado CF, Lucio PB. Elaboração do marco teórico: revisão de literatura e construção de uma perspectiva teórica. Metodologia de pesquisa. $3^{\mathrm{a}}$ ed. São Paulo: Mcgraw-hill; 2006. Cap. 4, p. 50-95.

8. Gomes GC, ErdmannAL, Oliveira PK, Xavier DM, Santos SS, Farias DH.Afamília durante a internação hospitalar da criança: contribuições para a enfermagem. Esc Anna Nery. 2014;18(2):234-40.

9. O'Connell K, Fritzeen J, Guzzetta CE, Clark AP, Lloyd C, Scott SH, et al. Family presence during trauma resuscitation: family members' attitudes, behaviors, and experiences. Am J Crit Care. 2017;26(3):229-39.

10. Buboltz FL, SilveiraA, Neves ET, Silva JH, Carvalho JS, Zamberlan KC. Percepção de familiares sobre sua presença ou não em situação de emergência pediátrica. Texto Contexto Enferm. 2016; 25(3):e0230015. 
Presença da família da criança nas situações de emergência e procedimentos invasivos: revisão integrativa

11. Silva JH, Buboltz FL, Silveira A, Neves EI, Portela JL, Jantsch LB. Permanência de familiares no atendimento de emergência pediátrica: percepções da equipe de saúde. Rev baiana enferm. 2017;31(3):e17427.

12. Shaw K, Ritchie D,Adams G. Does witnessing resuscitation help parents come to terms with the death of their child? A review of the literature. Intensive Crit Care Nurs. 2011;27(5):25362.

13. Ahmann E, Dokken D. Strategies for encouraging patient/family member partnerships with the health care team. Pediatr Nurs. 2012;38(4):232-5.

14. Tripon C, Defossez G, Ragot S, Ghazali A, Boureau-Voultoury A, Scépi M, et al. Parental presence during cardiopulmonary resuscitation of children: the experience, opinions and moral positions of emergency teams in France. Arch Dis Child. 2014;99(4):310-5.

15. Solà JA, Bravo SS, Cotanda CP, Maza VT, Cubells $\mathrm{CL}$. ¿ Ha aumentado la presencia de los padres durante los procedimientos invasivos en urgencias en los últimos años? An Pediatr (Barc). 2015;82(1):6-11.

16. Cruz AC, Angelo M. Cuidado centrado na família em pediatria: redefinindo os relacionamentos. Cienc. Cuid Saude. 2011;10(4):861-5.

17. Linhares DG, Siqueira JE, Previdelli IT. Limitação do suporte de vida em unidade de terapia intensiva pediátrica. Rev Bioét. 2013;21(2):291-7.

18. Strasburg AC, Pintanel AC, Gomes GC, Mota MS. Cuidado de enfermagem a crianças hospitalizadas: percepção de mães acompanhantes. Rev enferm. 2011;19(2):262-7.

19. Crowley L, Gallagher P, Price J. To stay or not to stay: children's nurses' experiences of parental presence during resuscitation. Nurs Child Young People. 2015;27(3):34-8.

20. Chapman R, Watkins R, Bushby A, Combs $S$. Assessing heath professional' perceptions of Family presence during resuscitation: a replication study. Int Emerg Nurs. 2013;21(1):1725.

21. Valadares MT, Mota JA, Oliveira BM. Cuidados paliativos em pediatria: uma revisão. Rev. Bioét. 2013;4(21):1-3.

22. Xavier DM, Gomes GC, Barlem EL, Erdmann AL. A família revelando-se como um ser de direitos durante a internação hospitalar da criança. Rev Bras Enferm. 2013;66(6):866-72.

23. Gomes GC, Oliveira PK. Vivências da família no hospital durante a internação da criança. Rev Gaúcha Enferm. 2012;4(33):165-71.

24. Sousa LD, Gomes GC, Silva MR, Santos CP, Silva BT. A família na unidade de pediatria: percepções da equipe de enfermagem acerca da dimensão cuidadora. Cienc Enferm. 2011;17(2):87-95.

25. Kingsnorth J, 0 `Connell K, Guzzetta CE, Edens JC, Atabaki S, Mecherikunnel A, et al. Family presence during trauma activations and medical resuscitations in a pediatric emergenc department: an evidence-based practice project. J Emerg Nurs. 2010;36(2):115-21.

26. Silva AF, Issi HB, Motta MG. A família da criança oncológica em cuidados paliativos: 0 olhar da equipe de enfermagem. Cienc Cuid Saude. 2011;10(4):820-7.
27. Xavier DM, Gomes GC, Santos SS, Lunardi VL, Pintanel AC, Erdmann AL. A família na Unidade de Pediatria: convivendo com normas e rotinas hospitalares. Rev Bras Enferm. 2014;67(2):181-6.

28. Lowry E. "It's just what we do": a qualitative study of emergency nurses working with wellestablished family presence protocol. J Emerg Nurs. 2012;38(4):329-34.

29. Porter J, Cooper SJ, Sellick K. Attitudes, implementation and practice of family presence during resuscitation (FPDR): a quantitative literature review. Int Emerg Nurs. 2013;21(1):26-34.

30. Mekitarian FF, Angelo M. Presença da família em sala de emergência pediátrica: opiniões dos profissionais de saúde. Rev Paul Pediatr. 2015;33(4):460-6.

31. Vavarouta A, Xanthos T, Papadimitriou L, Kouskouni E, lacovidou N. Family presence during resuscitation and invasive procedures: physicians' ande nurses' atitudes working in pediatric departments in Greece. Resuscitation. 2011;82(6):713-6.

32. Young KD. Observational study of Family member presence for pediatric emergency department procedures. Pediatr Emerg Care. 2014;30(7):449-52.

33. Jones BL, Parker-Raley J, Maxson T, Brown C. Understanding heath care professionals' views of family presence during pediatric resuscitation. Am J Crit Care. 2011;20(3):199-207.

34. González GS, Tomás RJ, Etxaniz JS. Presencia de familiares en los procedimientos realizados en urgencias de pediatríaopinión de la familia y de los profesionales. Emergencias. 2010;22(1):175-80.

35. Al-Mutair AS, Plummer V, Copnell B. Family presence during resuscitation: a descriptive study of nurses' attitudes from two Saudi hospitals. Nurs Crit Care. 2012;17(2):90-8.

36. Oczkowski SJ, Mazzetti I, Cupido C, Fox-Robichaud AE. The offering of family presence during resuscitation: a systematic review and meta-analysis. J Intensive Care. 2015;14(3):41.

37. Moreno CM, Castro CC, Cuesta AP, Gamero DB, Ferrer MMM. Presencia de los familiares durante la realización de procedimientos invasivos. An Pediatr (Barc). 2012;77(1):28-36.

38. Zavotsky KE, McCoy J, Bell G, Haussman K, Joiner J, Marcoux KK, et al. Resuscitation team perceptions of family presence during CPR. Adv Emerg Nurs J. 2014;36(4):325-34.

39. Feagan LM, Fisher NJ. The impact of education on provider attitudes toward Familywitnessed resuscitation. J Emerg Nurs. 2011;37(3):231-9.

40. Kantrowitz-Gordon I, Bennett D, Stauffer DW, Champ-Gibson E, Fitzgerald C, Corbett C. Facilitated family presence at resuscitation: effectiveness of a nursing student tookkit. Nurse Educ Today. 2013;33(10):1258-63.

41. Carroll DL. The effect of intensive care unit environments on nurse perpections of Family presence during ressuscitation and invasive procedures. Dimens Crit Care Nurs. 2014;33(1):34-9.

42. Edwards EE, Despotopulos LD, Carroll DL. Changes in provider perceptions of family presence during resuscitation. Clin Nurse Spec. 2013;27(5):239-44.

43. Porter JE, Cooper SJ, Taylor B. Family presence during resuscitation (FPDR): A survey of emergency personnel in Victoria, Australia. Australas Emerg Nurs J. 2015;18(2):98-105. 